2020-01-23

\title{
Epilepsy in People with Intellectual Disabilities
}

\author{
Shankar, Rohit
}

http://hdl.handle.net/10026.1/17224

$10.1093 / \mathrm{med} / 9780198794585.003 .0022$

Oxford University Press, USA

All content in PEARL is protected by copyright law. Author manuscripts are made available in accordance with publisher policies. Please cite only the published version using the details provided on the item record or document. In the absence of an open licence (e.g. Creative Commons), permissions for further reuse of content should be sought from the publisher or author. 


Abstract
It is well recognized that both Intellectual Disability (ID) and epilepsy individually have
higher rates of premature mortality. Thus, the two conditions in combination will be more
likely to lead to premature mortality than either individually. People with ID and epilepsy
have a higher likelihood of communication, psychiatric, behavioural, and drug sensitivity
problems that makes their treatment difficult. This is not a homogenous group. At one
end of the spectrum are patients with mild ID, 10-12\% of who have epilepsy and
treatment is mainly focused on areas like compliance and risky behaviours. At the other
end are patients with severe ID, up to $50 \%$ of who have epilepsy and treatment is more
focused on factors such as the co-morbidity, impact of medications, recognition of side
effects, treatment resistance, and informed consent. This chapter looks to collate and
provide an overview of epilepsy diagnosis and management and the current good practice
on its applicability to people with ID.
Keywords
intellectual disability, learning disability, autism, epilepsy, seizures




\title{
Chapter 22
}

\section{Epilepsy in People with Intellectual Disability}

\author{
Rohit Shankar and Matthew Walker
}

\section{Introduction}

Epilepsy is a potentially disabling, chronic and socially isolating condition. A diagnosis of epilepsy even now still carries a stigma. The individual and their family can be affected physically, psychologically and socioeconomically. Similarly, individuals with intellectual disability (ID), in broad terms a condition which occurs during the developmental period and leads to deficits in intelligence, overall development, and adaptive functioning skills or abilities, and their families and carers, experience a range of physical, social, and mental health issues. The co-existence of epilepsy and ID in an individual thus poses unique challenges (1).

Appropriate diagnosis and management of epilepsy are essential to reduce the considerable social impact, potential stigmatization, secondary handicap and low selfesteem compounded by social exclusion experienced by people with ID.

\section{Definitions}

Seizures are transient disturbed behaviour, emotional, motor or sensory symptoms or signs with or without an alteration in consciousness due to abnormal excessive or synchronous neuronal activity (2).

Epilepsy is the propensity to have recurrent unprovoked seizures. The International League Against Epilepsy (ILAE) $(2,3)$ state that epilepsy is a disease of the brain defined by any of the following conditions: 
1. At least two unprovoked (or reflex) seizures occurring greater than 24 hours apart

2. One unprovoked (or reflex) seizure and a probability of further seizures similar to the general recurrence risk (at least 60 per cent) after two unprovoked seizures, occurring over the next 10 years

3. Diagnosis of an epilepsy syndrome

Status Epilepticus is defined by the ILAE as a condition resulting either from the failure of the mechanisms responsible for seizure termination or from the initiation of mechanisms, which lead to abnormally, prolonged seizures (after time point t1). It is a condition which can have long-term consequences (after time point t2), including neuronal death, neuronal injury, and alteration of neuronal networks, depending on the type and duration of seizures (4). For convulsive status epilepticus, t1 is about 5 minutes and $\mathrm{t} 2$ is of the order of 30 minutes.

Sudden Unexpected Death in Epilepsy (SUDEP): SUDEP is the sudden and unexpected death of a person with epilepsy (PWE) when complete autopsy and toxicology does not identify a cause for death. Based on this definition, SUDEP is a diagnosis of exclusion. Definite SUDEP requires an autopsy to confirm no anatomical or toxicological cause. Probable SUDEP is applied when autopsy is not performed but the circumstances of death are otherwise very suggestive and possible SUDEP is when autopsy is not performed and there is a potential competing cause of death (5).

\section{Classifications}

\section{Classification of seizures}


Seizures are divided into two main types, and the terminology has been recently revised by the International League against Epilepsy:

1. Focal-onset Seizures - The seizures arise from brain networks that are limited to one hemisphere. These seizures are divided into focal seizures with or without loss of awareness (previously termed simple partial or complex partial seizures). They are then subdivided into those with motor (e.g. automatisms, clonic) or nonmotor onset (e.g. sensory, cognitive). Secondary generalization is now referred to as focal to bilateral tonicclonic.

2. Generalized-onset Seizures-Seizures that rapidly engage bilateral brain networks. Consciousness is impaired from the beginning, for example, absence, myoclonic, tonic-clonic, tonic, and atonic seizures. The latter seizure types are often associated with ID.

\section{Classification of epilepsy}

Similar to the classification of seizures, the ILAE has revised the classification of the epilepsies into (3):

1. The types of seizures - focal, generalized or combined focal and generalized.

2. The specific epilepsy syndrome (e.g. West syndrome, Lennox-Gastaut syndrome, Dravet syndrome etc.)

3. The presumed seizure aetiology-

- $\quad$ structural, genetic, infectious, metabolic, and/or immune or unknown 
Epilepsy is often caused by the same brain damage or maldevelopment that caused the ID. It is much more common in some conditions, particularly tuberous sclerosis. In people with Down syndrome, the onset of myoclonic epilepsy may herald the onset of dementia.

\section{Epidemiology}

\section{Epilepsy}

In the adult general population, epilepsy is the second commonest serious chronic neurological disorder after stroke (1). The National Institute for Health and Care Excellence (NICE) (4) stated that an accurate estimate of incidence and prevalence was difficult because of the problem in identifying and defining people who may have epilepsy. Epilepsy has been estimated to affect around 600,000 people in the UK. In addition, there will be further individuals, estimated to be 5-30 per cent, so approximately 100,000 people, who have been diagnosed with epilepsy, but in whom the diagnosis is incorrect. The incidence of epilepsy in high-income countries is estimated to be 50 per 100,000 per year (7) and the prevalence of active epilepsy in the UK is estimated to be 5-10 cases per 1000. Two-thirds of people with epilepsy can have their seizures completely controlled with anti-epileptic drugs (AEDs). Optimal management improves health outcomes and can also help to minimize other, often detrimental, impacts on social activities, education, and employment. However, a third of people with epilepsy do not achieve complete seizure control despite trying multiple medications.

\section{Epilepsy and intellectual disability}

Both epilepsy and ID may be caused by a range of pathological processes $(8,9,10)$. Among people known to ID services in the UK, the prevalence of epilepsy is 20-30 per 
cent and possibly higher in the residual populations of long-stay institutions (11). The prevalence of epilepsy in ID is between 22-26 per cent and increased with increasing level of ID $(12,13)$. The estimated prevalence for mild ID is around 10 per cent compared to 30 per cent for those with moderate, severe or profound ID (14). Two-thirds of people with ID and epilepsy are considered to show a poor response to anti-epileptic medication (13). People with ID and epilepsy have more physical impairments than those with ID but not epilepsy(12). However, whilst psychiatric or behavioural co-morbidity is common in people with ID, rates were not necessarily higher than in those with ID without epilepsy (12). It is associated with high healthcare costs and increased mortality (12). It was noted that in the UK convulsions and epilepsy were the most frequent cause of what were considered as potentially avoidable hospital admissions in people with ID, accounting for approximately 6,000 admissions a year, equivalent to 40 per cent of all emergency admissions for ACSCs in adults with ID (15). This is representative of the problem worldwide (16).

The co-existence of ID, drug refractory epilepsy, and neurological deficits are often associated with genetic/chromosomal abnormalities or with structural brain pathology (either damage or maldevelopment of the brain) (17). The known single gene mutations associated with ID and epilepsy is expanding; some of these are important for treatment strategies and so should always be considered. For example, SCN1A mutations are associated with Dravet syndrome, characterized by febrile and non-febrile seizures beginning in the first 12 months of life, episodes of status epilepticus, initial normal development, but intellectual decline in the second year of life. This syndrome can respond poorly to drugs that block sodium channels (e.g. lamotrigine, carbamazepine and 
phenytoin) (18). Tuberous sclerosis may respond well to vigabatrin (19). GLUT1

deficiency, which is associated with seizures in the first four months of life, dystonia (in particular exercise induced dyskinesia), and ID, may respond particularly well to a ketogenic diet (20). Supporting people with ID and epilepsy especially those with poorly controlled epilepsy requires high levels of competence and confidence in staff in community settings $(21,22)$.

Seizures in people with ID are commonly of multiple types and resistant to drug treatment $(23,24,25)$. This is especially true in severe and profound ID. Uncontrolled epilepsy can have serious negative consequences on both quality of life and mortality (26, 27). There is very poor understanding and evidence base supporting suitable prescribing in this vulnerable population (28).

The management of epilepsy is also particularly important because of the risk of SUDEP. The incidence of sudden death appears to be 20 times higher in patients with epilepsy compared to the general population, and SUDEP is the most important directly epilepsy-related cause of death (29). People with drug resistant epilepsy are particularly at risk of $\operatorname{SUDEP}(29,30)$. NICE (6) recommends that patients, carers and families need to be counselled using information tailored to the patient's relative risk of SUDEP. An evidence-based risk factor checklist to engage patients in such a person-centred discussion which includes people with ID has been developed (29). There is evidence that a bespoke service taking into account the current good practice for supporting people with ID and amalgamating it with good practice for managing epilepsy can reduce deaths in ID $(31,32)$. This is further highlighted by the recent ILAE special report, published in 2016 (33). It is important to mention that ID and epilepsy are also associated with various 
other syndromes, both epileptic (such as epileptic encephalopathies), whose discussion is beyond the scope of this chapter, and non-epileptic (Table 22.1).

\section{<insert table 22.1 here>}

\section{Diagnosis}

The diagnosis of epilepsy is clinical, and usually established by a specialist medical practitioner with training and expertise in epilepsy. Obtaining a good history of a patient with epilepsy and ID is very important and must cover information about when the epilepsy started and its progress over time(34). Detailed description of the seizures including duration, frequency, daytime/night time, abnormal movements, abnormalities of tone, any warning signs, events, and/or behaviours leading up to the seizure (pre-ictal), after the seizure (postictal), seizure hazards such head injuries, difficulty in breathing etc., triggers for the seizure, and any family history of neurological and psychiatric disorders is needed.

An eyewitness account of the episode is important and collateral information from family and carers is of critical importance. If appropriate, a request to have a video clip of the episode having ensured adequate security and protection is taken, not only to help the patient in the acute state but around the material collected can be a valuable asset for diagnosis. This can be obtained with patient's consent where an individual has the capacity to provide this, or through a formal best interest process using family/carers or an independent mental capacity advocate. It can be difficult to obtain sufficient information about early development in people with ID, especially in people with severe ID. 
Allied professional staff in day and residential services commonly attempt to classify seizures rather than describing them. They often use the out-dated terms 'grand mal' and 'petit mal'. 'Petit mal' is technically an earlier name for 'absences' and is all too often misused to cover any seizure or event that is not a typical tonic clonic seizure. Hence it is important that a description is obtained or recorded. When possible a video of the seizure by carers should be obtained as described above.

The clinical decision or diagnosis of an epileptic seizure should be based on the combination of the description of the attack and different symptoms. Diagnosis should not be based on the presence or absence of single features. If a definite diagnosis of epilepsy is not possible or cannot be clearly established, further investigations and/or referral to a tertiary epilepsy specialist should be considered. Follow-up should always be arranged (6). The diagnosis of epilepsy can be life changing, so there is a need for optimal diagnostic accuracy.

\section{Differential diagnosis}

Not all that shakes is epilepsy (35). The distinction is usually made on history (36). The differential diagnosis can include, syncope (e.g. vasovagal, orthostatic, cardiac arrhythmia), psychogenic states (e.g. panic attacks, dissociative disorders, affect disorders, psychosis), behavioural disorders (e.g. stereotypies, head banging, head rolling, body rocking obsessions, compulsions, self-injurious behaviour, self-stimulating behaviour), vascular pathology (e.g. migraine, transient ischemic attacks, transient global amnesia), sleep disorders (e.g. enuresis, nightmares, parasomnia, narcolepsy/cataplexy), metabolic (e.g. hypoglycaemia, hypernatremia, insulinoma, hypocalcaemia), migraine, movement disorders (e.g., paroxysmal dyskinesia), and toxic states (e.g. drugs, alcohol). 
The most common non-epileptic seizure is syncope caused by sudden decrease in perfusion of oxygenated blood throughout the brain(37). Table 22.2 provides the salient differences between seizures and syncope. Psychogenic non-epileptic seizures are discussed later in the chapter.

\section{<insert table 22.2 here>}

\section{Investigations}

Electroencephalography (EEG) through the use of electrodes placed on the scalp records electrical changes produced by the superficial cerebral cortex. It helps in identifying the location of any epileptic focus. A normal EEG between seizures does not rule out epilepsy. Unless an actual seizure is captured on the recording, an EEG can only support a clinical diagnosis of epilepsy and should not be used in isolation. 'Epileptiform' activity on an EEG does not necessarily mean that the diagnosis is epilepsy; even 0.5 per cent of aircrew have an abnormal EEG (38) and this percentage is much higher in people with ID, psychiatric disease, and/or brain damage, especially when on anti-psychotics. Most people with ID will be able to co-operate with an EEG but would need to have it explained to them in terms they understand, and consented appropriately (including explaining the risks of seizures with activating procedures such as sleep deprivation, photic stimulation, or hyperventilation). Steps to examine mental capacity and consenting are provided in pathway 1 (see box 22.1).

\section{$<$ Box 22.1>}

Sleep deprived EEG, repeat EEG, long-term video, or ambulatory EEG increase the EEG yield. Video telemetry, which combines EEG and video recording is invaluable in determining whether an identified behaviour is epileptic or not. 
Neuroimaging of the brain routinely includes Computed Tomography (CT) Scanning and Magnetic Resonance Imaging (MRI). CT scanning is readily available and can provide information on brain symmetry and on large potentially epileptogenic lesions like infarction or tumours. It is particularly useful where there are calcified abnormalities and skull changes. However, CT is insufficiently sensitive to use as definitive imaging in most people with epilepsy. MRI has essentially replaced CT as the imaging of choice for epilepsy because of its sensitivity and specificity in identifying structural lesions that could be the origin of epileptic discharges. However, MRI is not always widely available, making CT sometimes still the appropriate initial investigation especially during emergencies. With careful preparation many people with ID can have an MRI scan, but some will need heavy sedation or a general anesthetic and in such instances, the yield of MRI and the clinical impact have to be weighed against the risk of such a procedure.

Pathway 1 (Box 22.1) highlights the processes which need to be considered in consenting people with ID.

Electrocardiogram (ECG) is an important investigation to exclude possible heart conditions that could resemble epilepsy or when an individual is reported to have experienced blackouts and falls of unknown cause.

Laboratory studies are useful in excluding metabolic or toxic causes of seizures (e.g. hypernatremia, hypoglycaemia, drugs).

\section{Management}

As antiepileptic medication is the most important and relevant method to ensure control of seizures. It is important to get the treatment right in this vulnerable group to prevent 
avoidable harm. There is very little evidence-based research for AED prescribing in people with ID (28).

People with epilepsy and ID should have the same access to a specialist (epilepsy) service and have a comprehensive care plan agreed (6). As already mentioned, epilepsy is not only more common in people with ID but can also be more difficult to treat, so both the regular treatment and focus on the emergency treatment of acute seizures in this group is important (39). People with ID are at higher risk of being on concomitant medications that lower the seizure threshold, increasing their risk of seizure occurrence (40).

There are multiple outcomes that might be the focus of treatment. The principle aims of management are for the patient to be seizure free and free from any adverse medication effects. However, often this cannot be fully realized, especially given the difficulties in diagnosis and higher potential of treatment resistance. In such a situation it would be good to help the patient make informed choices on what are realistic outcomes. Where the patient is lacking the mental capacity to make or participate in these choices, a best interest meeting (as per the Mental Capacity Act 2005 in the UK) with key stakeholders should be held to provide guidance and goals for seizure management, using similar principles outlined in pathway 1 .

The current management can comprise of pharmacological treatment in the form of antiepileptic drugs (AED), vagus nerve stimulation (VNS), and resective surgery. The ketogenic diet has been shown in randomized controlled trials to be effective in children with refractory epilepsy, but this class of evidence is, at present, lacking in adults. 
The management approach must be centred on the needs and wishes of the person with epilepsy and take into account their experiences and social context. Education about epilepsy is important for all those with epilepsy, as well as those who support them. Video, pictures and photographs can be used to support education, which should be directed towards families and care staff as well as the person with epilepsy. Language, sequencing and memory difficulties may contribute to poor adherence to a treatment regime, and thus these need to be considered actively when planning a treatment strategy.

It must be kept in mind that a 'one size fits all' approach using 'easy read' or communication via videos etc. has its limitations, and possible use of communication experts such as speech and language therapists can be useful. People with ID and epilepsy have typically experienced very little control of their own lives. Resentment over this may also lead to difficulties, especially with adherence to complex drug treatment.

It is also worth bearing in mind that 'improvement' in seizures does not always translate as an 'improvement' for the individual and their carers. There are not only individual expectations but carer expectations to contend with. A principal example is the emergence of behavioural and cognitive side effects to AED treatment. This is usually reported by family or carers. A Cochrane Review (41) found that the majority of studies in this field typically used no or nonreplicable measures of behavioural exacerbation, were uncontrolled, and were mostly retrospective in nature. A systematic review reported no difference in rates of behavioural problems between individuals with ID who have epilepsy and those who do not (12). Specifically regarding AEDs, research indicates that 
some AEDs may have both positive and negative behavioural side effects. Research in this area is, however, significantly limited.

When assessing for presence of a potential behavioural side effect, careful consideration needs to be given to the following questions:

1. Is it a side effect that has been described for the drug (Table 22.3 $)$ ?

2. Would this side effect be expected in someone with the same nature and degree of ID? This information is limited due to lack of evidence at the present time.

3. Could it be that the improvement in seizure control has enabled the person with epilepsy to be better able to express their opinion?

4. Is this a problem that is due to the personality, cognitive deficit, and/or social skills of the individual as opposed to side effects of the medication?

5. Is this the result of drug interactions due to polypharmacy, as occurs in many people with ID? (42).

6. How accurate is the information from carers?

7. Would therapy be useful for behavioural management?

\section{<insert table 22.3 here>}

An enquiry into the family or social dynamics of the individual, the nature and type of behaviour and the involvement of behavioural, communication, and occupational therapists would be helpful in developing a coherent formulation. Attempts should be made to retain the medication if it has been efficacious, and withdrawal should only be considered if there is a clear indication of an association of the side effect to behaviour or mental state. Often reduction of dose rather than withdrawal of the medication may be sufficient to address such side effects. 
Behaviour and its manifestations have a pervasive impact on people with ID and epilepsy. Both ID and epilepsy vary by nature and severity with little evidence on how they associate. Any person with an ID, epilepsy, and behavioural disorder should be provided with multidisciplinary care (33).

\section{Pharmacological treatment}

AEDs are the mainstay treatment for epilepsy. Pathway $2(\overline{B o x} 22.2)$ lists the considerations needed to prescribe to people with ID. However, the level of evidence of individual AEDs to specific groups of people with ID is limited (28). Treatment should be individualized, starting with a single AED (monotherapy) wherever possible at a low initial dose with slow titration, unless rapid control of seizures is required (6). Should this be unsuccessful, another AED (monotherapy) should be tried. This is when the primary monotherapy is unsuccessful or the patient develops adverse effects, the second AED is introduced and built up to a therapeutic or tolerable dose before the first AED is slowly withdrawn. Combination therapy should only be considered if monotherapy fails. Though some AEDs work best against particular seizure types, several AEDs may have to be tried $(28,43,44)$. There is also a significant lack of evidence of the use of individual AEDs in different subsets of ID.

\section{<insert box 22.2>}

A popular choice are benzodiazepines (e.g. clonazepam, clobazam, clonazepam, diazepam and midazolam), which are used as both rescue medication and as an effective add-on treatment in refractory epilepsy, especially prevalent in people with an ID (28). Clobazam in particular is recognized as being especially useful as intermittent rescue treatment, given in short courses to break up clusters of seizures or to provide short-term 
break to help develop more robust treatment strategies. Clobazam is considered appropriate to use regularly as second line or adjunct therapy for all major seizure types, and is of particular value in refractory epilepsy (28). Tolerance is a major issue, particularly in ID populations, although it is speculated that around 30 per cent of PWE on clobazam could continue without encountering long-term tolerance (28). The potential negatives of tolerance include the distress of changing medication and the need to reduce the drug slowly. The other risk is that significant numbers of PWE and ID find themselves on various benzodiazepines to handle behaviour, mood, or anxiety. There needs to be awareness of the overall 'benzodiazepine load' by clinicians who are prescribing and monitoring clobazam for seizures.

Benzodiazepine treatments have been criticized for potential adverse side effects, including cognitive impairment in long-term use in both general and ID populations, and as a result are favoured more for use as rescue medication (28). There are no definitive studies or guidelines to manage treatment using this group of drugs.

At present it is advised that people with an ID, especially those susceptible to balance problems, are not prescribed phenytoin. It is also not recommended for long-term use, as it can lead to marked cognitive impairment or symptoms and signs of cerebellar disease (28). Preventing phenytoin intoxication and subsequent phenytoin-induced encephalopathy remains dependent upon very careful monitoring of people and frequent monitoring of drug levels (28). This would prove difficult in people with ID given their distress potential to recurrent venepuncture and difficulties to fully comprehend the need for monitoring. The available literature highlights the complexity of using phenytoin in ID populations and the lack of systematic evaluation. 
Gabapentin and lamotrigine are broadly considered safe treatment for epilepsy in people with an ID (28). Lamotrigine has been investigated in various studies in people with ID and found to be broadly of positive benefit. However, the study designs and selection of ID populations prevent robust conclusions of side effect and efficacy profiles. A single study looked at topiramate and found that it is generally well tolerated and did not have a negative impact upon behaviour. This study concluded that topiramate reduced seizure rates in patients with epilepsy and ID without compromising quality of life (28).

Sodium valproate has been widely used as a broad-spectrum treatment option anticonvulsant drug for over 40 years with a good safety profile (28). Some research has demonstrated that due to this it is recommended for use in patients with seizures which are challenging to classify and thus those with treatment-resistant epilepsy with an ID may be more responsive to valproate treatment (28). Limitations for the use of valproate in the general population are that although it is first choice it is teratogenic and often causes significant weight gain. Furthermore, in some people (in particular those with urea cycle deficits or mitochondrial disease), valproate therapy can increase plasma ammonia, sometimes leading to encephalopathy and a paradoxical worsening of seizures.

Studies of tolerability of AEDs such as ethosuximide, carbamazepine, sodium valproate, eslicarbamazepine, lacosamide, retigabine, zonisamide, levetiracetam, and perampanel in ID populations are extremely limited. To date, the tolerability and efficacy of these AEDs has not been evaluated in any suitable fashion amongst ID populations. The UK Ep-ID research Register is a National Institute of Health Research adopted project whose design is of a retrospective cohort real world study looking at outcomes of 
tolerability and efficacy of various AEDs in people with ID based on their nature and severity of ID (28). A recent enquiry into perampanel by the Register has highlighted that people with moderate to profound ID are less likely to drop out possibly due to their inability to report or communicate subjective side effects and higher rates of seizure improvement due to higher rates of retention and compliance (45).

A problem uncommonly encountered in ID populations is of pregnancy when on AEDs. A recent study identified 217 pregnancies to mothers with recorded ID of 245,007 births 1970-1989 in Oxford (population: 850,000). No major differences were seen in offspring of mothers with ID (28).

No studies have looked at teratogenic effects of AEDs specifically in mothers with ID and epilepsy. It would be expected that the impact of AEDs on pregnancy and their children would be no different in mothers with ID as in the general population. There could, however, be additional confounders such as genetic disorders to which the foetus might be more vulnerable. Given the significantly low numbers expected of mothers with ID on AEDs (statistically less than one/year for one million populations) there would be an expectation that such a pregnancy and post birth would be managed with highest levels of monitoring and surveillance ${ }^{(28)}$.

Patients on AEDs need to be monitored for adverse effects. All AEDs by necessity cross the blood brain barrier. All therefore have the potential to produce adverse effects on alertness, cognition, and mental state. These effects may be particularly pronounced in people with ID (46).

Evidence, such as it is, indicates that lamotrigine should be considered first-line therapy for focal epilepsy and valproate should be considered first-line for generalized 
epilepsy (47). However, in view of its teratogenic potential, valproate should be avoided in women of child-bearing age, unless the woman is using appropriate contraception. This might be an academic issue in women in the moderate to profound ID range. However, there is now an increase in pregnancies in women with mild ID. For this population the complexity of teratogenic risk may prove beyond their comprehension (48) and these women would benefit from proactive management, including ensuring that valproate is not used.

Blood monitoring does not need to be routinely done unless clinically indicated (e.g. breakthrough seizures or unexpected occurrence of side effect) or to manage phenytoin doses; nevertheless, baseline blood levels for some drugs are helpful when people are on stable doses. AEDs should be introduced and withdrawn slowly with the aim of achieving the lowest effective dose. It is dangerous to stop AEDs suddenly, because this may precipitate status epilepticus even when the AED has not apparently been effective.

People with ID and their carers may not understand the importance of adhering to a treatment regime. A simple regime, the use of pictures, and close liaison with the pharmacist all help. The use of pictures to communicate with people with ID can help (49). Healthcare professionals have a role in educating patients, carers, and family members in understanding epilepsy, the rationale for treatment, reducing the stigma attached, and developing positive relationships (6).

If a patient is seizure free for a period of two years whilst on AED then withdrawal of AED can be considered. This should however be discussed with patient, their carers, and/or family where possible, and actions done with the best interest of the 
patient in mind. Withdrawal of the AED should be monitored and under the guidance of a specialist (6). Withdrawal of AEDs, even when optimally undertaken, can be associated with re-emergence of seizures. In people with ID a decision to withdraw AEDs need to be taken alongside consideration of the likelihood of the benefits the medication is offering versus the fact that people with ID can be more likely to be treatment resistant, thus possibly being at higher risk of a relapse.

\section{Status epilepticus}

Individuals with ID are more likely to develop status epilepticus (50). The mortality risk is also increased for this patient group. The treatment of status epilepticus is considered a medical emergency. Treatment should be given if the individual has a prolonged (convulsive) seizure that lasts for five minutes or more or if seizure occurs three or more times in an hour (6). Treatment options available in the community include buccal midazolam (first-line treatment) and rectal diazepam (if midazolam is not suitable). The administration of rectal diazepam in the community raises dignity issues. Other medications can be prescribed within the hospital setting. Depending on the individual's personalized care plan and their response to their rescue treatment an ambulance needs to be called if seizure carries on after the administration of treatment and/or if there are concerns about their breathing, airway, or other vital signs.

Carers and family need to be appropriately trained in the administration of these rescue medications. Standards of training however vary across the UK, with potential consequences that could be serious for the individual patient given the risk of brain damage and mortality (51). Shortly there is to be released new national guidance on training and good practice of administering midazolam as an update from the previous 
Joint Epilepsy Committee guidance (52). There is a development to also have a

standardised e-test to ensure safe and consistent practice.

\section{Vagus nerve stimulation (VNS)}

Although AEDs remain the main treatment for intractable epilepsy, a significant proportion of people with ID will respond poorly to AEDs, with up to 40 per cent on polytherapy but poor seizure control $(46,53)$, which means that alternatives need to be considered. VNS is indicated for use as an adjunctive therapy in reducing the frequency of seizures in adults who are refractory to AED and are not suitable for resective surgery. There is some evidence that VNS can be effective in multiple seizure types, including drop attacks, which carry a high morbidity (54). VNS is a relatively safer surgical option for patients with ID (55). One should be mindful of short- and long-term side effects, including rare presentations such as an impact on heart conduction (56). Where there are communication and capacity issues, in-depth consenting processes need to be addressed prior to surgery.

\section{Resective surgery}

Epilepsy in people with ID can be refractory to AEDs and so can be potentially suitable for resective surgery. The main role of epilepsy surgery is to achieve seizure freedom or a significant reduction in seizure frequency, without producing adverse cognitive or psychological effects (57). ID is not a contraindication to surgery (33), and surgery can be considered even when multiple lesions are present, as in tuberous sclerosis. The risks to the patient of on-going refractory epilepsy and the impact that it could have on quality of life should be considered when contemplating the possibility of epilepsy surgery. Issues 
such as mental capacity and ability to make informed choices on the advantages, and disadvantages of surgery can sometimes be challenging.

\section{Psychological impact}

Cognitive activity is affected by the underlying brain pathology, by the effects of repeated interruptions in consciousness, and by AEDs. All tend to reduce cognitive function. This may lead to sedation and poor motivation, or it may be expressed as irritability, impulsiveness, and disinhibition (58).

Repeated disruption of consciousness interrupts memory and learning. This is a particular problem in 'absence' seizures, where the seizures are brief and may be unrecognized, but are often frequent, especially in children. A person with undiagnosed nonconvulsive status epilepticus may resemble someone with severe ID or autism, and can sometime explain deterioration in cognition or behaviour. An EEG is necessary for diagnosis of this under-diagnosed condition. When their epilepsy is treated, their whole demeanour may change dramatically.

Epilepsy can have a negative effect on self-esteem. It is a hidden disability, but one that can be internally stigmatizing (59).

Many AEDs, particularly but not exclusively, vigabatrin, can produce behaviour problems and even psychosis. As previously mentioned, valproate can be associated with a metabolic encephalopathy, presenting as worsening of seizures or deterioration in mental capacity.

Psychological disturbance may occur before, during, or after a seizure. People with focal seizures commonly experience a warning or aura, which may take the form of a particular emotional state or a hallucinatory experience. Some seizures, particularly 
focal seizures affecting the temporal or frontal lobes result in the person behaving in a bizarre and stereotyped way without full awareness, though they may exhibit apparent conscious behaviour. After a seizure many people sleep, some have headaches, and many are irritable or confused (60). Post-ictal psychosis can also rarely occur, usually after a lucid interval of hours to days, and lasts from days to weeks. This is usually associated with disordered thought, paranoia, and aggressive behaviour.

An EEG should be considered when there is any enduring change in mental capacity, state, or behaviour or unexplained deterioration to exclude non-convulsive status epilepticus.

Detailed discussion on behavioural issues, ID, and epilepsy has been presented earlier in the chapter.

\section{Social impact}

Watching a person have a convulsive seizure can be frightening. People unfamiliar with epilepsy can think the person is dying. For some relatives/carers, and some people with epilepsy, this fear persists. There are also real risks of serious injury, for example by drowning during a bath or falling downstairs or in front of traffic. Services are afraid of litigation. It is not surprising that people with epilepsy and ID are sometimes offered more protection than they need. Risk management is an important aspect of epilepsy but could come at the cost of access to community or impact on quality of life (for example stopping swimming or hydrotherapy). One needs to be mindful, especially if the concerned individual has limited ability to make informed choices, that the risk management needs to be tempered by pragmatism, and the least restrictive frameworks of care balanced against possible risk needs to be established. 
People with epilepsy are usually allowed to drive if they have been free of seizures for a year as per UK law (61). However, it is important that if this is an issue the latest advice is taken from the GP and/or the DVLA. Most people with ID do not drive. However transport can still be a problem, especially if the individual is on a rescue medication protocol. Practical issues such as lack of an escort or frequent public transport can impact heavily on the individual's quality of life. Seizures further limit access to employment, leisure, and sporting activities (62). They therefore contribute to poverty and social isolation. This in turn contributes to a sense of powerlessness and low selfesteem.

In some cultures, epileptic seizures are seen as evidence of demonic possession or of infection. This can mean that people are reluctant to touch or share cutlery with anyone who has seizures. People with ID from such cultures can be highly vulnerable to prejudice and discrimination.

Rescue medication administration, such as buccal midazolam or rectal diazepam can pose particular difficulties. It is useful in the prevention of status epilepticus in vulnerable individuals and can be given by specially trained, but otherwise unqualified people. It can enable people with ID to avoid frequent in-patient admissions. However staff may be reluctant to use it. They may fear injuring the person or that there could be accusations of sexual abuse especially with rectal diazepam use.

An individually based person-centred assessment at regular intervals of the real risks of particular activities and treatments will often allow the person to lead a much fuller life. Such a plan needs to consider suitability of the residential placement and access to activities and services suitable to the individual's needs and recreation. An 
epilepsy care plan, centred on the person with epilepsy and involving everyone with a responsibility to care for them, is helpful. Such a plan should form part of the Health Action Plan recommended by the UK White paper, Valuing People Now (2009) (63).

\section{Risk management}

This will usually involves assessing the patient's level of risk and depends on the individual, their environment, frequency, and severity of epilepsy (64). Professionals need to be aware of the higher risk held by individuals with ID and epilepsy and that these be discussed with the individual, their families, and/or carers (6). It is also recommended that a risk assessment takes place and includes bathing and showering, preparing food, using electrical equipment, managing prolonged or serial seizures, the impact of epilepsy in social settings, SUDEP, and the suitability of independent living (6, $65,66)$.

The SUDEP and seizure safety checklist is a 10 minute evidenced-based risk communication tool which is available free to download and use $(29,31,32,67)$.

Various national and international charities issue excellent leaflets on various activities and epilepsy, for example, sport, leisure, employment, and computers. The emphasis should be on having strategies in place to prevent injury from occurring if the person has a seizure, rather than restricting their activities $(65,66)$.

\section{Psychogenic non-epileptic seizures (PNES)}

The misdiagnosis rate of epilepsy is considered to be around 25-30 per cent. It is considered that around 90 per cent of the misdiagnosis is PNES when seizure presentation is co-related with EEGs (68). To diagnose PNES can be difficult, as it usually involves overturning an existing diagnosis. It is made more problematic as there 
are no diagnostic tests or cluster of symptoms that are completely reliable. It is a diagnosis of exclusion.

The patient's history may suggest the diagnosis. Certain clinical features and presenting symptoms should raise the suspicion that seizures may be psychogenic rather than epileptic (Table 22.4).

<insert table 22.4 here>

Resistance to antiepileptic drugs (AEDs) is usually the first clue, though epilepsy in people with ID can be difficult to treat. Approximately 80 per cent people with PNES have been treated with AEDs before the correct diagnosis is made. A psychogenic aetiology should be considered when AEDs have no effect on the reported frequency of seizures.

Specific psycho-social triggers such as stress, fibromyalgia, chronic pain, chronic fatigue, somatization, and environmental situations need to be considered. PNES rarely occurs in sleep and is more frequent in the presence of other people (audience). A psychosocial history with evidence of maladaptive behaviours or associated psychiatric diagnoses should raise the suspicion of PNES. A mental status exam, highlighting soft signs such as the presenting general demeanour of the person and their carers, appropriateness of the level of concerns, over dramatization, hysterical features and in particular looking to 'scapegoat' seizures as explanation for relationship dysfunction is helpful. In people with ID, especially those with mild ID, where there could be a degree of suggestibility, it is important to assess if their 'seizures' are facilitating a 'sickness role' which stands to the benefit of the individual and/or carer.

It is not uncommon for a detailed seizure history to reveal characteristics not consistent with epileptic seizures. An episode captured on EEG would provide significant 
insight to the diagnosis however this could be difficult to achieve, especially in people with ID. A resistance to gainfully make inroads towards validating the diagnosis such as refusing to bring a video sample or an alternative carer are also soft signs suggestive of PNES.

It is expected that the episodes would have been investigated as seizures.

The following categories are suggested for a diagnosis of PNES (69):

- Documented PNES — confirmed by clinical history plus EEG video monitoring

- $\quad$ Clinically established PNES — defined by clinical history, clinician witness, and EEG recording of habitual events without video

- $\quad$ Probable PNES - determined by clinical history, clinician witness of video or live events, and a normal EEG

- $\quad$ Possible PNES - relies on patient's self-report of clinical events and a normal EEG

The main obstacle to effective treatment is effective delivery of the diagnosis. Delivering the diagnosis of PNES, especially to a person who has received a diagnosis of an organic illness such as epilepsy could be challenging. The patient reaction could be adversarial in the form of disbelief, denial, anger, and hostility. Patients who accept their diagnosis and follow through with therapy are more likely to experience a successful outcome; therefore, patient education is crucial. It is important that the patient recognize that the diagnosis is incidental and change in symptom profile would require alternative strategies. In people with ID a communication specialist might be of benefit, to help provide the information in a manner the patient can comprehend. 
Treatment of PNES varies and can include psychotherapy and use of adjunctive medications to treat coexisting anxiety or depression. Evidence exists that indicates that Cognitive Behaviour Therapy (CBT) significantly reduces psychogenic seizure frequency compared to standard medical care (70). This was further reinforced in a study that showed CBT and sertraline (an antidepressant) together provided better outcomes for seizure control than the sertraline only group (71). However a recent Cochrane review concluded that there is little reliable evidence to support the use of any treatment, including CBT, in the treatment of non-epileptic seizures (72).

The counter issue of misdiagnosis of epilepsy by not diagnosing epilepsy needs to be considered. A systematic review showed that between 32-38 per cent of people with ID were diagnosed as not having epilepsy or as having nonepileptic events (73). The main reason for misdiagnosis was the misinterpretation of behavioural, physiological, syndrome-related, medication-related, or psychological events by parents, paid carers, and health professionals. The review indicates high levels of non-epileptic events which have the potential to be misdiagnosed as epileptic events in people with and without ID. The occurrence of seizures may be both over-estimated and under-recognized. People may experience a combination of epileptic and non-epileptic events, and in some cases it may not be possible to reach a diagnosis. The knowledge of family members, support workers and a range of health staff also affects whether events are correctly diagnosed as epileptic or not. These issues are not unique to people with ID. However, the findings do suggest that people with ID are likely to face additional barriers to receiving an accurate diagnosis. The review establishes that cognitive issues, behavioural problems, 
communication difficulties, motor problems, and side effects of medication experienced by people with ID may be misinterpreted as epileptic events.

\section{Seizures, intellectual disability and challenging behaviour}

Epilepsy and behavioural disturbances are closely associated and have multiple possible associations. This is a complex area which has been poorly researched.

1. Seizures can cause behavioural disturbance as part of the seizure phenomenology.

2. Seizures can lead to brain damage which can result in personality change and maladaptive behaviours.

3. Seizures can co-exist with mental illness which can then have a symbiotic effect of influencing behaviour.

4. Medication especially first generation and third generation AEDs can have a negative impact on behaviour.

5. Successful treatment of seizures can still leave a damaged or underdeveloped personality which would lack social skills to cope and live in their environment. This can result as challenging behaviour.

It is important that any challenging behaviour assessment be person centred and include the diagnosis and impact of seizures in the formulation. It is important to separate primary associations such as direct organicity and medication impact on behaviour from secondary links where the behaviour is a function of frustration, communication, poor coping mechanisms, or habituation to get specific responses. The cause and effect needs to be understood. It is critical that the overall best interest of the individual be considered and not have a piecemeal approach of treating only the epilepsy or the behaviours 
separately. Management strategies should be on the lines identified in recent good practice documents(74).

\section{Conclusion}

Managing epilepsy in people with ID is not only challenging but requires a diverse skill set. Complexities encountered in the routine management of epilepsy in the general population such as compliance, alcohol, and recreational drug issues, driving concerns, and pregnancy are more limited in the ID population. However, there is higher representation of communication deficits, lack of informed decision-making, physical and mental health comorbidity, side-effect sensitivity and higher levels of total drug prescribing, which bring their own challenges. Lately there has been the development of good practice guidance reports from the Royal College of Psychiatrists UK on the delivery of epilepsy care (75) for this vulnerable population and on prescribing (74). Further, in the field of epilepsy there is a rapid growth of technology (76), understanding of influence of genetics and improved characteristics of seizures and risk $(77,78,79)$. It is hoped that such documents and the attention they bring will help stimulate more research to improve understanding of this complex area as research in ID and epilepsy still remains significantly lower than other clinical areas (80).

\section{Case study 1}

Mary, a 46-year-old who has moderate ID with seizures in remission for over five years and on sodium valproate $2000 \mathrm{mgs} / \mathrm{day}$ and haloperidol presented with five month history of lassitude, tiredness, falls, and minor confusion having had three episodes of respiratory infections requiring antibiotic care. There was a recurrence of seizures with the infection which was perceived by the general practitioner to be secondary to the high temperature 
occurring with the infection. Initially there was consideration of her going through menopause. On being reviewed, full blood count among other bloods was requested. It revealed thrombocytopenia. Sodium valproate was then gradually reduced to $1500 \mathrm{mg} /$ day and a re-test of full blood count in three months' time showed full recovery. Along with it her problems of tiredness, infections, seizures, and falls stopped

\section{Case study 2}

Jason is 20 and has mild ID, transient mood disorder, and Down syndrome. He was diagnosed with epilepsy and started on levetiracetam without full consideration of his psychological issues at his local hospital. The levetiracetam with each increase in dose as advised by the BNF improved his seizures but worsened his mood and behaviours. $\mathrm{He}$ became aggressive and violent and was commenced on risperidone by his GP which had an impact of improving his behaviours but again causing a worsening of his seizures. Eventually when referred to a specialist in ID and epilepsy, sodium valproate was commenced and titrated and on stabilization both the risperidone and levetiracetam withdrawn gradually one by one.

\section{Case study 3}

Rachel has a Rett's syndrome and severe to profound ID. She presented with brief apnoea attacks with no associated symptomology to aid diagnosis of whether these attacks were of seizure origin. She would not co-operate to have an ambulatory EEG. As there is seizure association of $>90$ per cent with Rett's a trail of AED was attempted. Rachel's apnoea attacks receded with each dose increase and went to complete cessation.

\section{Multiple choice questions}




\section{Questions}

1. The prevalence of epilepsy in people with ID is approximately
A. 10 per cent
B. 20 per cent
C. 30 per cent
D. 40 per cent

2. Which of these statements about SUDEP is false?
A. SUDEP is more common in general population than people with ID
B. SUDEP is linked to having frequent generalized seizures
C. Duration of poorly treated epilepsy is associated with increased SUDEP risk
D. QTc prolongation and other cardiac irregularities are associated with higher risk of SUDEP

3. Which one of these statements on investigations is true?
A. EEGs are essential in people with ID as they can definitely confirm presence of seizures
B. It is important to investigate irrespective of the distress caused to the patient
C. A 'best interest' process to investigations is essential for an individual with ID who cannot consent and could get distressed by attempts to do so
D. It is not important to consider a head MRI in a new patient presenting with episodes suggestive of seizures

4. Which of these should be avoided in managing epilepsy in people with ID? 
A. Slow titration of AED possibly at half or quarter of the suggested rates for general population

B. If reported behavioural side effects immediately stop the AED

B. While swapping ideally build up an alternate AED then withdraw the other

D. The risks of having more than two regular AEDs could significantly outweigh the benefits of their positive effects

5. Which of these combinations is considered to be a beneficial one?
A. Phenytoin-Carbamazepine
B. Carbamazepine-Lamotrigine
C. Clobazam-Zonisamide
D. Lamotrigine-Sodium valproate

\section{Answers}

1. B. Robertson et al (https://www.ncbi.nlm.nih.gov/pubmed/26076844) in their systematic review identified the pooled estimate for epilepsy in people with ID from 38 studies was 22.2 per cent (95\% CI 19.6-25.1). A population-based study by McGrother et al. (https://www.ncbi.nlm.nih.gov/pubmed/16782360) highlighted prevalence of epilepsy in people with ID to be 26 per cent

2. A. In the overall epilepsy population, mortality rates are approximately 2.5 -fold higher than in the general population Cockerell et al.

(https://www.ncbi.nlm.nih.gov/pubmed/7934347), and Hauser et al. (https://www.ncbi.nlm.nih.gov/pubmed/7398606). A US population-based study 
by Ficker et al. (https://www.ncbi.nlm.nih.gov/pubmed/9818844) showed SUDEP rates in the epilepsy population exceeded the expected rate of sudden death in the general population by 24 times. Sillanpaa et al.

https://www.nejm.org/doi/full/10.1056/NEJMoa0911610) in their cohort study of following 245 children with epilepsy for 40 years reported 24 per cent mortality of which 30 per cent were classified as SUDEP.

3. C. While all reasonable attempts need to be taken to encourage the individual to participate in investigations to help identify and assess for seizures consideration needs to be given to the potential for distress and the benefits one gains from an investigation by subjecting a non-capacitous individual through the process. If the person requires to be sedated for a brief EEG then the risks of the sedation, the impact of the sedation on the EEG activity, and the potential lack of any obvious seizure activity at the time of investigation and overall utility should be weighed in balance to the potential iatrogenic trauma to the individual.

4. B. Abrupt withdrawal of AED can lead to significant increased risk of SUDEP and higher recurrence rates of seizures.

5. D. There is a significant synergistic drug interaction with valproate (valproate is an enzyme inhibitor, lamotrigine clearance is reduced, and thus lamotrigine levels are higher. There is evidence to suggest valproate and lamotrigine combine positively and synergistically offering improved results in refractory epilepsy; Brodie et al. https://www.ncbi.nlm.nih.gov/pubmed/9127723) and Pisani et al. https://www.ncbi.nlm.nih.gov/pubmed/10448829).

\section{References}


1. Prasher VP, Kerr MP. Epilepsy and Intellectual Disabilities. 2008 Springer.

2. Fisher SF, Acevedo C, Arzimanoglou A et al A practical clinical definition of epilepsy. Epilepsia. 2014;55(4):475-482.

3. Scheffer IE, Berkovic S, Capovilla G et al. ILAE classification of the epilepsies: Position paper of the ILAE Commission for Classification and Terminology. Epilepsia. 2017;58(4):512-521.

4. Trinka E, Hesdorffer D, Rossetti AO et al. Definition and classification of status epilepticus - report of the task force on classification of status epilepticus. Epilepsia. 2015;56(10):1515-1523.

5. Nashef L, So E, Ryvlin P et al. Unifying the definitions of sudden unexpected death in epilepsy. Epilepsia. 2012;53(2):227-233.

6. The National Institute for Health and Care Excellence (NICE). The epilepsies: The diagnosis and management of the epilepsies in adults and children in primary and secondary care. 2012 NICE clinical guideline 137 guidance.nice.org.uk/cg137.

7. Sander JW, Shorvon SD. Epidemiology of the epilepsies. J Neurol Neurosurg Psychiatry. 1996;61:433-443.

8. Forsgren L, Edvinsson S-O, Blomquist HK et al. Epilepsy in a population of mentally retarded children and adults. Epilepsy Res. 1990;6:234-238.

9. Bowley C, Kerr M. Epilepsy and intellectual disability. J Intellect Disabil Res. 2000;44(5):529-543.

10. Lhatoo SD, Sander JWAS. The epidemiology of epilepsy and learning disability. Epilepsia. 2001;42(Suppl. 1):6-9. 
11. Bell GS, Sander JW. The epidemiology of epilepsy: The size of the problem. Seizure. 2001;10(4):306-314.

12. Robertson J, Hatton C, Emerson E et al. Prevalence of epilepsy among people with intellectual disabilities: A systematic review. Seizure. 2015;29:46-62.

13. McGrother CW, Bhaumik S, Thorp CF et al. Epilepsy in adults with intellectual disabilities: Prevalence, associations and service implications. Seizure. 2006;15:376-386

14. Robertson J, Baines S, Emerson E et al. Service responses to people with intellectual disabilities and epilepsy: A systematic review. J Appl Res Intellect Disabil. 2015 doi:10.1111/jar.12228.

15. Glover G, Evison F. Hospital admissions that should not happen. Improving Health and Lives: Learning Disabilities Observatory; 2013 ([Available from:https://www.improvinghealthandlives.org.uk/publications/1169]).

16. Balogh R, Brownell M, Ouellette-Kuntz H, Colantonio A. Hospitalization rates for ambulatory care sensitive conditions for persons with and without an intellectual disability: A population perspective. Journal of Intellectual Disability Research. 2010;54:820-832.

17. Busch RM, Najm I, Hermann B, Eng C. Genetics of cognition in epilepsy. Epilepsy and Behavior: E\&B. 2014;0:297-306.

18. Catterall WA. Sodium Channel Mutations and Epilepsy. In: Noebels JL, Avoli M, Rogawski MA, et al., editors. Jasper's Basic Mechanisms of the Epilepsies [Internet]. 4th edition. Bethesda (MD): National Center for Biotechnology Information (US); 2012. 
19. Curatolo P, Verdecchia M, Bombardieri R. Vigabatrin for tuberous sclerosis complex. Brain Dev. 2001; 23(7):649-653.

20. Klepper J, Scheffer H, Leiendecker B et al. Seizure control and acceptance of the ketogenic diet in GLUT1 deficiency syndrome: A 2- to 5-year follow-up of 15 children enrolled prospectively Neuropediatrics. 2005;36(5):302-308.

21. Kerr M, Linehan C, Thompson R et al. A white paper on the medical and social needs of people with epilepsy and intellectual disability: The Task force on intellectual disabilities and epilepsy of the international league against epilepsy. Epilepsia. 2014;55:1902-1906.

22. Thompson R, Kerr M, Glynn M et al. Caring for a family member with intellectual disability and epilepsy: Practical, social and emotional perspectives. Seizure. 2014;23:856-863.

23. Amiet C, Gourfinkel-An I, Bouzamondo A, Tordjman S, Baulac M, Lechat P, et al. Epilepsy in autism is associated with intellectual disability and gender: evidence from a meta-analysis. Biological Psychiatry. 2008;64:577-582.

24. Branford D, Bhaumik S, Duncan F. Epilepsy in adults with learning disabilities. Seizure. 1998;7:473-477.

25. Matthews T, Weston N, Baxter H, Felce D, Kerr M. A general practice-based prevalence study of epilepsy among adults with intellectual disabilities and of its association with psychiatric disorder, behaviour disturbance and carer stress. Journal of Intellectual Disability Research. 2008;52:163-173.

26. Kerr M, Bowley C. Evidence-based prescribing in adults with learning disability and epilepsy. Epilepsia. 2001;42(Suppl. 1):44-45. 
27. Kerr M, Bowley C. Multidisciplinary and multiagency contributions to care for those with learning disability who have epilepsy. Epilepsia. 2001; 42(Suppl. 1):55-56.

28. Doran Z, Shankar R, Keezer MR, et al. Managing anti-epileptic drug treatment in adult patients with intellectual disability: A serious conundrum. Eur J Neurol. 2016;23:1152-1157.

29. Shankar R, Cox D, Jalihal V et al. Sudden unexpected death in epilepsy (SUDEP): Development of a safety checklist. Seizure. 2013;22:812-817.

30. Hesdorffer DC, Tomson T, Benn E et al. Commission on Epidemiology. Subcommission on mortality combined analysis of risk factors for SUDEP. Epilepsia. 2011;52(6):1150-1159.

31. Shankar R, Jalihal V, Walker M et al. A community study in Cornwall UK of sudden unexpected death in epilepsy (SUDEP) in a 9-year population sample. Seizure. 2014;23(5):382-385.

32. Shankar R, Walker M, McLean B et al. A step to modify and prevent SUDEP. The validity of risk factors in the SUDEP and Seizure Safety Checklist: A case control study. Journal of Neurology DOI 10.1007/s00415-016-8203-3.

33. Kerr M, Linehan C, Brandt, $\mathrm{C}$ et al. Behavioral disorder in people with an intellectual disability and epilepsy: A report of the Intellectual Disability Task Force of the Neuropsychiatric Commission of ILAE. Epilepsia Open. 2016. doi:10.1002/epi4.12018.

34. Bowley C, Kerr M. Epilepsy and intellectual disability. J Intellect Disabil Res. 2000;44(Pt 5):529-543. 
35. Rothner AD. 'Not everything that shakes is epilepsy'. The differential diagnosis of paroxysmal nonepileptiform disorders. Cleveland Clinic Journal of Medicine. 1989;56 Suppl Pt 2:S206-S213.

36. Hopkins A, Shorvon S, Cascino G. Epilepsy. 2 ed. 1995 London: Chapman \& Hall.

37. Crompton D, Berkovic S. The borderland of epilepsy: Clinical and molecular features of phenomena that mimic epileptic seizures The Lancet Neurology, 2009, Volume 8, Issue 4, 370-381.

38. Gregory RP, Oates T, Merry RTG. EEG epileptiform abnormalities in candidates for aircrew training. Electroenceph Clin Neurophysiol. 1993;86:75-77.

39. Pellock JM, Morton LD. Treatment of epilepsy in the multiply handicapped. Ment Retard Dev DR. 2000;6: 309-323.

40. http://www.rcpsych.ac.uk/pdf/FR_ID_09_for_website.pdf (accessed 22/10/2016).

41. Beavis J, Kerr M, Marson AG. Pharmacological interventions for epilepsy in people with intellectual disabilities. Cochrane Database Syst Rev.

$$
\text { 2007;(3):CD005399. }
$$

42. Camfield PR, Bahi-Buisson N, Trinka E. Transition issues for children with diffuse cortical malformations, multifocal postnatal lesions (infectious and traumatic), and Lennox-Gastaut and similar syndromes. Epilepsia. 2014;55:2428.

43. Feely M. Drug treatment of epilepsy. BMJ. 1999;318 (9 January):106-109.

44. Livanainen M, Alvarez N. Drug treatment of epilepsy in people with intellectual disability. JIDR. 1998;42 Suppl 1:iv. 
45. Shankar R, et al. Presented at ECE 2016, Prague, Czech Republic, September 1115.

46. Espie CA, Gillies JB, Montgomery J M. Antiepileptic polypharmacy, psychosocial behaviour and locus of control orientation among mentally handicapped adults living in the community. J Ment Defic Res.1990;34:351-360.

47. Marson, A et al. The SANAD study of effectiveness of valproate, lamotrigine, or topiramate for generalised and unclassifiable epilepsy: An unblinded randomised controlled trial. The Lancet, 2007, Volume 369, Issue 9566, 1016-1026.

48. Porter E et al., Developing the pregnancy support pack for people who have a learning disability British Journal of Learning Disabilities, 2012, 40: 310-317.

49. Hollins, S. Books Beyond Words: Telling the Whole Story in Pictures. 2003. Comment [A5]: AQ: Year?

Available: http://www.intellectualdisability.info/how-to../books-beyond-wordstelling-the-whole-story-in-pictures. Last accessed 22/10/2016.

50. Pellock JM, Morton LD. reatment of epilepsy in the multiply handicapped. Ment Retard Dev D R 6. 2000:309-323.

51. Rohit Shankar Testing Competency in Delivering Epilepsy Protocol Medication. Advances in clinical neuroscience and rehabilitation: ACNR 06/2014; 14(2):31.

52. Joint Epilepsy Committee. Buccal Midazolam Guidelines. 2014. Available: http://www.jointepilepsycouncil.org.uk/resources/buccal_midazolam.html. Last accessed 22/10/2016.

53. Singh BK, Towle P O. Antiepileptic drug status in adult outpatients with mental retardation. Am J Ment Retard. 1993;98: 41-46.

54. Zamponi et al. Seizure 2011;20(6):468-474. 
55. Prasher VP. Furlong E, Weerasena L. Vagus Nerve Stimulation Therapy: An Intellectual Disabilities Perspective. In: Prasher VP Kerr MP. Epilepsy and Intellectual Disabilities. 2008. London: Springer. Pp. 109-128.

56. Shankar R, Olotu V, Cole N, Sullivan H, Jory C. Case report: Vagal nerve stimulation and late onset asystole. Seizure. 2008;22 (4):312-314.

57. Nicolson, A. Resective Surgery for Patients with Epilepsy and Intellectual Disabilities. In: Prasher V Kerr, M. Epilepsy and Intellectual Disabilities. 2008. London: Springer. Pp. 129-151.

58. Espie CA, Pashley AS, Bonham KG, Sourindhrin I, O'Donovan M. The mentally handicapped person with epilepsy: A comparative study investigating psychosocial functioning. Journal of Mental Deficiency Research 1989;33:123135.

59. Scambler G. Stigma and disease: changing paradigms. Lancet. 1998;352(9133):1054-1055.

60. Fenwick P. (1995). Psychiatric Disorder and Epilepsy. In: Hopkins A, Shorvon SD, Cascino G, editors. Epilepsy. London: Chapman Hall, pp. 453-502.

61. Shorvon SD. Epilepsy and driving. BMJ. 1995;310:885-886.

62. Scambler G, Hopkins A. Generating a model of epileptic stigma: The role of qualitative analysis. Soc Sci Med. 1990;30(11):1187-1194.

63. Department of Health. Valuing People Now: A new three year strategy for people with learning disabilities. Department of Health, London, 2009. 
64. International Association of the Scientific Study of Intellectual Disabilities (IASSID). Clinical guidelines for the management of epilepsy in adults with an intellectual disability. Seizure 2001;10:401-409.

65. Forjuoh SN, Guyer B. Injury prevention in people with disabilities. BMJ. 2001;322:940-941.

66. Besag F. Lesson of the week: Tonic seizures are a particular risk factor for drowning in people with epilepsy. BMJ. 2001;322:975-976.

67. https://sudep.org/checklist accessed 22/10/2016.

68. Benbadis SR. Errors in EEGs and the misdiagnosis of epilepsy: Importance, causes, consequences, and proposed remedies. Epilepsy Behav. 2007;11(3):257262.

69. LaFrance WC Jr, Baker GA, Duncan R, Goldstein LH, Reuber M. Minimum requirements for the diagnosis of psychogenic nonepileptic seizures: A staged approach.A report from the International League Against Epilepsy; Nonepileptic Seizures Task Force. Epilepsia. 2013;54(11):2005-2018.

70. Goldstein LH, Chalder T, Chigwedere C, Khondoker MR, Moriarty J, Toone BK et al. Cognitive-behavioral therapy for psychogenic nonepileptic seizures: A pilot RCT. Neurology. 2010;74(24):1986-1994.

71. LaFrance WC Jr, Baird GL, Barry JJ, Blum AS, Frank Webb A, Keitner GI et al. Multicenter pilot treatment trial for psychogenic nonepileptic seizures: A randomized clinical trial. JAMA Psychiatry. 2014;71(9):997-1005.

72. Martlew J, Pulman J, Marson AG. Psychological and behavioural treatments for adults with non-epileptic attack disorder. Cochrane Database of Systematic 
Reviews. 2014, Issue 2. Art. No.: CD006370. DOI:

10.1002/14651858.CD006370.pub2.

73. Chapman Melanie et al. The misdiagnosis of epilepsy in people with intellectual disabilities: A systematic review. Seizure 20 2011:101-106.

74. Prescribing Antiepileptic Drugs for people with epilepsy and Intellectual Disability. Royal College of Psychiatrists College Report 206. 10/2017.

75. Management of Epilepsy in people with Intellectual Disability. Royal College of Psychiatrists College Report 203. 05/2017.

76. Mclean B, Shankar R Hanna J et al. SUDEP — Measures to reduce risk Pract Neurol. 2017;17:13-20

77. Watkins L, Shankar R, Sander JW. Identifying and mitigating. Sudden Unexpected Death in Epilepsy (SUDEP) risk factors. 2018. Expert Rev Neurotherapeutics. DOI: 10.1080/14737175.2018.1439738.

78. Watkins L Shankar R. Reducing the risk of Sudden Unexpected Death in Epilepsy. Curr Treat Options Neurol. 2018;20: 40.

\section{https://doi.org/10.1007/s11940-018-0527-0.}

79. Young C, Shankar R, Henley W, et al. SUDEP and seizure safety communication: Assessing if people hear and act. Epilepsy Behav. 2018. https://doi.org/10.1016/j.yebeh.2018.06.040.

80. Shankar R, Rowe C, Van Hoorn A et al. Under representation of people with epilepsy and ID in research. PLOS One 10.1371/journal.pone.|0198261. 


\section{Pathway 1 Consenting process for investigations for an individual with ID designed using the Mental Capacity Act UK 2005}

1. Presume the person to have capacity to make an informed decision.

2. If the individual has ID, be sensitive to the possibility of the person needing more support in receiving and processing the information.

3. Consider utilizing a communication specialist (speech and language therapist) and suitable communication medium, that is, visual, tablet, social story etc.

4. Ensure there are no other deficits such as hearing or visual deficits.

5. Environment needs to be stress free and the patient should be relaxed.

6. If needed arrange for a visit to the centre. People with ID and their carers appreciate having the opportunity to make a reconnaissance pre-visit to the hospital EEG and scan rooms and meet key personnel.

7. Ask for feedback on understanding of key points and feedback on visit.

8. Be flexible to provide reasonable adjustments at the centre, for example, having a double appointment space.

9. Little issues such as parking availability and an identified co-ordinator such as a liaison nurse who knows the setting being there to support the person from start to finish could be invaluable.

10. Be prepared to call off the process if the individual becomes distressed as the trauma can have long-standing influence on the person with ID and negatively affect their future access to health care. 
11. If the person lacks capacity to make an informed decision on the investigations then a formal best interest meeting would need to be held.

12. The best interest meeting would need to involve all key stakeholders including family, carers, patient's GP, social worker, and other relevant people such as key workers and hospital staff who conduct the procedure. If there is no family member or friend who can represent the patient an independent mental capacity advocate needs to be sought. Care should be taken that even if the patient lacks capacity their wishes, if any, on the situation can be considered suitably.

13. The best interest meeting needs to consider how relevant and important the investigation is to the individual in the context of the patient's compliance and distress potential to it. If it is felt absolutely necessary but there is a significant likelihood of patient distress the possibility of doing the process under sedation or general anaesthetic (GA) needs exploring. The risk of the GA or sedation should be factored into the relevance of the assessment.

14. An attempt to explain the final decision to the patient needs to be made.

15. Proactive planning for the procedure such as organization of ambulances, minimizing delay, ensuring medication is given etc. must be care planned along with relevant risk assessments and contingency plans.

16. Throughout the pathway it is imperative good records be kept and information communicated clearly between all parties. 
Box 22.2

\section{Pathway 2 Considerations to prescribing in people with ID}

1. Attempt to ensure good clinical practice for investigation and diagnosis of epilepsy is implemented.

2. Each person with ID is different and it is important to gauge the level of understanding of the individual to make informed choices around benefits and risks of medication.

3. Consider providing more clinic time for appointments for people with ID.

4. Attempt to provide individuals and carers with suitable information at a level they can comprehend-easy read literature etc.

5. If the individual lacks the mental capacity to make informed choices consult with key stakeholders in the patient's best interest and provide relevant information on medication especially impact and side effects.

6. Define seizure improvement to the individual and agree on a method of monitoring and reporting.

7. Look to make medication choices based on evidence base as described in the paper, syndrome diagnosis, comorbidities, and other current medication.

8. Gain feedback appropriately including impact on mood, behavior, and social activity

9. If noted resistance to medication consider alternatives including the possibility of the movements/seizure description being due to other 
conditions which mimic seizure presentations such as tics, stereotypes in people with ID, and autism.

10. Arrange for structured reviews and feedback.

Table 22.1

Common ID syndromes associated with epilepsy

\begin{tabular}{|c|c|}
\hline ID Syndrome & $\begin{array}{l}\text { Percentage of Patients Diagnosed With } \\
\text { Epilepsy }\end{array}$ \\
\hline Autism & $30 \%$ \\
\hline $\begin{array}{l}\text { Autism with severe intellectual disability and } \\
\text { cerebral palsy }\end{array}$ & $67 \%$ \\
\hline Degenerative disorders & $70 \%$ \\
\hline Rett syndrome & $70-90 \%$ \\
\hline
\end{tabular}

Table 22.2

Differences between seizures and syncope

\begin{tabular}{|c|c|c|}
\hline & Seizures & Vasovagal Syncope \\
\hline History of seizures & Yes & No \\
\hline Onset & Sudden & Sudden \\
\hline Prodromal mood changes & Associated & $\begin{array}{l}\text { Unusual, but presyncopal features are } \\
\text { common including light-headedness, } \\
\text { sweating, clamminess, nausea, and/or } \\
\text { blurred vision }\end{array}$ \\
\hline Precipitating factors & $\begin{array}{l}\text { Rare. Flashing lights (rare, } \\
\text { less than } 5 \% \text { ) }\end{array}$ & $\begin{array}{l}\text { Unpleasant physical or emotional } \\
\text { stimuli (pain, fright etc.) }\end{array}$ \\
\hline Incontinence & Associated & Unusual \\
\hline Pulse rate & $\begin{array}{l}\text { Often elevated but may be } \\
\text { normal }\end{array}$ & Slow at onset, later rapid and weak \\
\hline Respiratory rate & $\begin{array}{l}\text { Depends on phase and type } \\
\text { of seizure }\end{array}$ & Normal \\
\hline Tone & Increased & Floppy \\
\hline Tongue bitten or scarred & Associated & Unusual \\
\hline Appearance & Cyanosed or normal & Pale and sweaty \\
\hline Recovery phase & Post ictal & Rapid recovery on assuming the \\
\hline
\end{tabular}




\begin{tabular}{|l|l|l|}
\hline & & supine position \\
\hline
\end{tabular}

Table 22.4

Psychogenic non-epileptic seizures

\begin{tabular}{|c|c|c|}
\hline & Seizure & Non Epileptic Attack \\
\hline Precipitating cause & Rare & Common: stress and emotion \\
\hline Location & $\begin{array}{l}\text { Can happen in sleep and when } \\
\text { alone }\end{array}$ & $\begin{array}{l}\text { Occur in wakefulness, but can } \\
\text { happen from sleep. Often occurs } \\
\text { in company }\end{array}$ \\
\hline Onset & Usually short & Variable \\
\hline Aura & Usually stereotyped_-various & $\begin{array}{l}\text { Fear, panic, altered mental state, } \\
\text { and dissociation }\end{array}$ \\
\hline Duration & Few minutes & Variable \\
\hline Consciousness & $\begin{array}{l}\text { Complete loss in generalized } \\
\text { tonic clonic seizures; may be } \\
\text { incomplete in complex partial } \\
\text { seizures }\end{array}$ & $\begin{array}{l}\text { Is variable and often inconsistent } \\
\text { with the considered seizure type }\end{array}$ \\
\hline Movement & $\begin{array}{l}\text { Consistent with seizure type, } \\
\text { synchronous, small amplitude } \\
\text { jerks }\end{array}$ & $\begin{array}{l}\text { Asynchronous, opisthotonous, } \\
\text { flailing of limbs, pelvic thrusting }\end{array}$ \\
\hline Speech & $\begin{array}{l}\text { Grunt at onset; word } \\
\text { automatisms, cry }\end{array}$ & Unintelligible, semi-voluntary \\
\hline Response to stimulation & $\begin{array}{l}\text { None in generalized tonic-clonic } \\
\text { may respond in complex partial } \\
\text { and postictal }\end{array}$ & $\begin{array}{l}\text { Often reacts and this may } \\
\text { terminate episode }\end{array}$ \\
\hline Incontinence & Common & Sometimes \\
\hline Injury & $\begin{array}{l}\text { Tongue bite (usually side), fall, } \\
\text { directed violence rare }\end{array}$ & $\begin{array}{l}\text { Tongue biting is usually tip and } \\
\text { rarely draws blood } \\
\text { Carpet burns are not uncommon } \\
\text { Directed violence not uncommon }\end{array}$ \\
\hline Recovery & $\begin{array}{l}\text { Dependent of seizure type-few } \\
\text { minutes with or without } \\
\text { confusion } \\
\text { Possible tiredness and need to } \\
\text { sleep }\end{array}$ & $\begin{array}{l}\text { May be rapid or very prolonged. } \\
\text { Tearfulness often occurs }\end{array}$ \\
\hline
\end{tabular}

Reproduced from National Guideline Centre (2012) Epilepsies: diagnosis and management, Clinical guideline 137. 
Published by the National Guideline Centre at The Royal College of Physicians, 11 St Andrews Place, Regent's Park, London, NW11 4LE. Copyright @ NGC. Reproduced by permission.

Table 22.3

\begin{tabular}{|l|l|l|}
\hline Anxiety/nervousness & $\begin{array}{l}\text { Pschosis/psychotic } \\
\text { symptoms }\end{array}$ & Aggression/hostility \\
\hline Clobazam $^{*+}$ & (Carbamazepine) & (Carbamazepine) $^{*}$ \\
\hline Clonazepam & Clobazam $^{*+}$ & Clobazam $^{*}$ \\
\hline Eslicarbazepine acetate & Clonazepam & Clonazepam $^{+}$ \\
\hline Gabapentin & Eslicarbaepine acetate & Ethosuximide \\
\hline Levetiracetam & Etho suximide & Gabapentin \\
\hline Oxcarbazepine & Lacosamide & Lacosamide \\
\hline Perampanel & Levetiracetam & Lamotrigine \\
\hline Topiramate & Topiramate & Levetiracetam \\
\hline Vigabatrin & Vigabatrin & Perampanel \\
\hline Zonisamide & Zonisamide & Pregabalin \\
\hline & & Topiramate \\
\hline & & Valproate \\
\hline & & Vigabatrin \\
\hline & & Zonisamide \\
\hline
\end{tabular}

*†Especially in paediatric or elderly population. Brackets indicate rare/very rare $(<0.1 \%)$. Bold indicates common $(\geq 1 \%$ to $<10 \%$ ). Remainder are uncommon $(\geq 0.1 \%$ to $<1 \%)$ or rates are not given (clobazam, clonazepam).

Source data from the Summary of Product Characteristics.

Electronic Medicines Compendium (eMC) https://www.medicines.org.uk/emc/ (Accessed 12/06/2018). (C) Datapharm. 